Prepared for the U.S. Department of Energy

Under Contract DE-AC05-76RL01830

\title{
FY 2011 Miniature Spherical Retroreflectors - Final Report
}

NC Anheier

BE Bernacki

HA Qiao

September 2011

Pacific Northwest

NATIONAL LABORATORY

Proudly Operated by Battelle Since 1965 


\title{
DISCLAIMER
}

This report was prepared as an account of work sponsored by an agency of the United States Government. Neither the United States Government nor any agency thereof, nor Battelle Memorial Institute, nor any of their employees, makes any warranty, express or implied, or assumes any legal liability or responsibility for the accuracy, completeness, or usefulness of any information, apparatus, product, or process disclosed, or represents that its use would not infringe privately owned rights. Reference herein to any specific commercial product, process, or service by trade name, trademark, manufacturer, or otherwise does not necessarily constitute or imply its endorsement, recommendation, or favoring by the United States Government or any agency thereof, or Battelle Memorial Institute. The views and opinions of authors expressed herein do not necessarily state or reflect those of the United States Government or any agency thereof.

\author{
PACIFIC NORTHWEST NATIONAL LABORATORY \\ operated by \\ BATTELLE \\ for the \\ UNITED STATES DEPARTMENT OF ENERGY \\ under Contract DE-AC05-76RL01830 \\ Printed in the United States of America
Available to DOE and DOE contractors from the Office of Scientific and Technical Information,
P.O. Box 62, Oak Ridge, TN 37831-0062; ph: (865) 576-8401 fax: (865) 576-5728
email: reports@adonis.osti.gov

\footnotetext{
Available to the public from the National Technical Information Service, U.S. Department of Commerce, 5285 Port Royal Rd., Springfield, VA 22161 ph: (800) 553-6847 fax: $(703) 605-6900$ email: orders@ntis.fedworld.gov online ordering: http://www.ntis.gov/ordering.htm
} 


\section{FY 2011 Miniature Spherical Retroreflectors - Final Report}

NC Anheier

BE Bernacki

HA Qiao

September 2011

Prepared for

the U.S. Department of Energy

under Contract DE-AC05-76RL01830

Pacific Northwest National Laboratory

Richland, Washington 99352 



\section{Executive Summary}

This report concludes a multi-year NNSA Office of Nuclear Nonproliferation Research and Development (NA-22) project that investigated a promising remote sensing concept based on miniature spherical retroreflectors. The goal of the Miniature Spherical Retroreflector project was to develop optical retroreflector designs, fabrication methods, and sensing concepts to aid in the detection of nuclear proliferation and other chemical vapor or radiation signatures. Miniature spherical retroreflectors offer a distinct advantage, since they can be dispersed over a broad area, yet remain extremely difficult to detect except by the laser transceiver. Optimized designs offer improved standoff performance due to their large optical cross section and the reduced retroreflected beam divergence compared to opportune specular reflections from remote objects in the field.

Prior research efforts demonstrated this concept's promise, but lacked rigorous optical design consideration and were ultimately limited by the fabrication bottleneck that has inhibited development and deployment of this wide-angle, optically interrogated remote sensing concept. The PNNL research plan was organized to develop mid-wave infrared (MWIR) and long-wave infrared (LWIR) miniature retroreflector designs, explore practical methods of fabrication, and finally evaluate integrated sensing concepts. An intensive numerical simulation effort was undertaken that resulted in optimized step-index optical designs for mid-infrared applications. Optical ray trace modeling was performed to better understand the geometrical dependencies of the miniature spherical retroreflector application. We adopted and advanced the concept of optical cross section, a metric that provides relative performance comparisons between different retroreflector designs. Our cross section analysis demonstrated that our step-index design provided $90 \%$ of the range capacity of an ideal spherical index design.

Encouraged by the performance potential, our group investigated fabrication approaches for the stepindex design. Two potential methods were evaluated for step-index retroreflector fabrication. The first fabrication method was based on a Flow Focusing micro-nozzle. This processing begins by heating the glass in a platinum crucible and allowing it to flow through an orifice at the bottom of the crucible. The micro-nozzle injector is centered in the crucible orifice and pressurized with inert gas. A funnel-shaped lens of liquid is produced by the pressure drop across the orifice. By controlling the flow rate of liquid glass and the micro-nozzle pressure, a steady stream of small glass spheres can be produced. Integration of a second nozzle shell allows the simultaneous formation of a concentric shell, resulting in the desired step-index design. Our group investigated this approach by modifying the Pacific National Northwest Laboratory (PNNL) draw tower with a micro-nozzle assembly. Thermal visco-elastic properties of chalcogenide glass were studied and then the micro-nozzle process was evaluated. While the Flow Focusing micro-nozzle investigation demonstrated promising results and potential for mass production of miniature step-index retroreflectors, it became clear that the development costs were becoming prohibitive within the scope of the project budget, so we concentrated our efforts toward a compressionmolding approach.

PNNL developed technical specifications for a custom compression molding press and partnered with a commercial company to develop a press system specifically designed for our investigation. The project focused on evaluating the molding behavior of candidate infrared glass materials and developing molding

processes for the retroreflector elements. The thermal expansion mismatch between the core and cladding materials caused the clad retroreflectors to fracture upon cooling from molding to room temperature and was identified as a major challenge in both Super Vidron-BK7 and IG3-ZnS material systems. This is an 
unfortunate consequence of the clustering of physical properties of a moldable glass; namely, the low glass transition temperature that permits molding at reasonable temperatures along with a correspondingly high coefficient of thermal expansion. Research efforts continued to develop a solution to the thermal expansion mismatch problem. We introduced a half-clad step-index design that has significant performance and fabrication advantages. The half-clad design promised fabrication simplification, reduced stress encountered during cooling, and consequently increased yield. Unfortunately we determined that molding yield remained poor due to the difficulty of molding the extremely thin optical chalcogenide shells.

Ultimately we were not able to overcome the optical material properties that limited the viability of fabrication by compression molding. Our current investigation instead evaluated a mid-infrared retroreflector based on a Cat's Eye design that could be fabricated using traditional optical lens manufacturing techniques and manual assembly. In addition, a client approved project scope change also allowed evaluation of quantum dot retroreflector layers. To demonstrate the performance of the Cat's Eye approach, custom retroreflector optical elements were fabricated by a commercial optical manufacturer, and then was assembled and tested in our lab. The additional retroreflector sensing task evaluated retroreflector sensing films based on nanocrystalline materials called quantum dots. To test the feasibility of this concept, index $n=2$, ball retroreflectors were coated with quantum dots as an example sensing layer. In a darkened laboratory, the laser-excited photoluminescence was easily observed by eye at 46-meter standoff distance. 


\section{Acknowledgments}

The authors would like to thank PNNL staff members Steve Miller, Marvin Warner, and Cynthia Warner for their stimulating discussions regarding nanocrystal $\mathrm{LiF}_{2}$ synthesis and assistance with quantum dot preparation. 



\section{Acronyms and Abbreviations}

CS

CTE

FEA

GLS

HMOX

IR

NA-22

OSL

PNNL

SR

TTL cross section

coefficients of thermal expansion

finite element analysis

gallium lanthanum sulfide

heavy metal oxide

infrared

NNSA Office of Nuclear Nonproliferation Research and Development optically stimulated luminescence

Pacific National Northwest Laboratory

Strehl ratio

tagging, tracking, and locating 



\section{Contents}

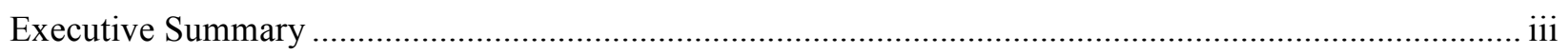

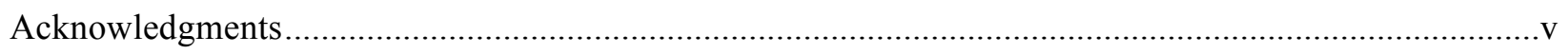

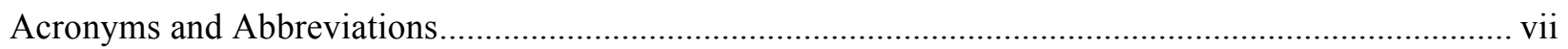

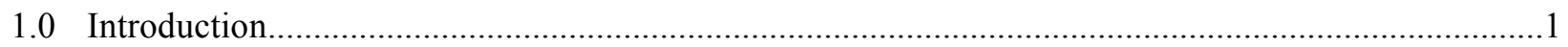

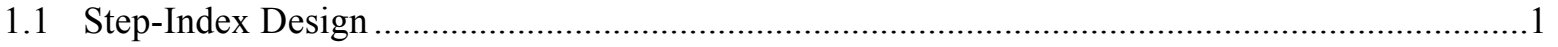

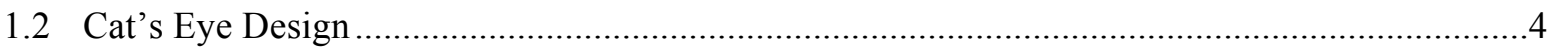

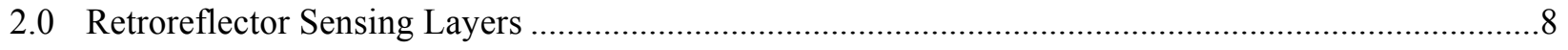

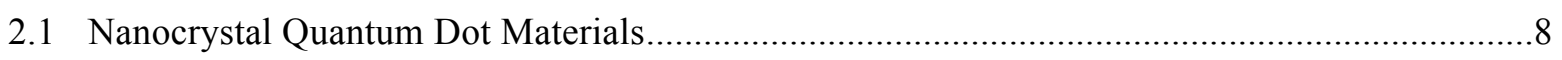

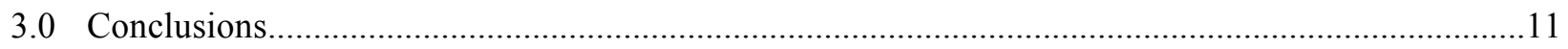

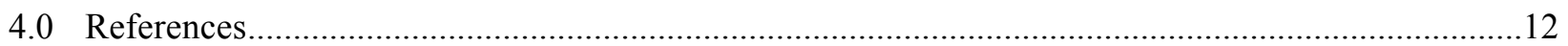




\section{Figures}

1 Comparison of the Step Index Profile and an Optimized Spherical Gradient Profile for a

7.744-mm-Diameter Sphere and the Optimized Step-Index Retroreflector with Incident and

Reflected Ray Trajectories

2 The SR Dependence as a Function of Entrance Pupil Diameter as the Clad Ball Design is Optimized), and the Dependence of SR on Entrance Pupil Diameter is Used to Compute the CS that Reveals that the Peak CS is Achieved for an Entrance Pupil Diameter of $\sim 4 \mathrm{~mm}$.....

3 The Retroreflected Beam SR Versus Input Beam Diameter for Three Different Retroreflector Designs: Homogeneous Ball, Clad or Step-index Sphere, and Gradient Index Variants

4 Cross Sections for the Strehl Ratios of the Four Designs Shown Earlier in Figure 3

5 Cat's Eye Retroreflector Utilizing a Si and Ge Hemisphere and SR versus Input Beam Diameter for the Cat's Eye Design

6 On-axis SR as a Function of Ge Hemisphere Thickness and Off-axis SR as a Function of Ge Hemisphere Thickness

7 On-axis CS as a Function of Ge Hemisphere Thickness and Off-axis CS as a Function of Ge Hemisphere Thickness

8 Anti-reflection Coated $8 \mathrm{~mm}$ Diameter Silicon Hemisphere that Serves as the Cat's Eye Input and the Rear, Gold-coated $4 \mathrm{~mm}$ Diameter Germanium Hemisphere

9 The Spatial Intensity Map and the Angular Intensity Distribution of the Return at 10 Meters, Indicating Promising Cat's Eye Retroreflector Performance ......

10 Cat's Eye Retroreflector Return at 0.6 Meters and at 1.2 Meters ....................................................

11 The Spatial Intensity Map and the Angular Intensity Distribution of the Return at 1 Meter ...............8

12 Lattice Structure of Bulk Materials that Leads to Broad Continuum Emission in Luminescent Materials and Nanocrystal Quantum Confinement that Leads to Bright and Narrow Bandwidth Luminescence.

13 Homogeneous Index $n=2$ Spherical Retroreflector with an Applied CdSe/ZnS Quantum Luminescent Layer and Extremely Strong Retroreflected Luminescent Emission Easily Seen about 46 Meters Away 


\subsection{Introduction}

PNNL conducted a systematic optical modeling effort to study and compare retroreflector designs with respect to available optical materials (Anheier et al. 2005-2008). The optical Strehl ratio (SR) and cross section figures of merit were used to compare the performance of candidate optical designs (Anheier et. al. 2007). Further modeling was conducted to assess the relationships between standoff range and retroreflector diameter and cross section. Analysis showed that small diameter retroreflectors have very high optical link loss, since the net loss scales by retroreflector diameter to the $4^{\text {th }}$ power. Optical retroreflection efficiency is also important, but extracting ideal performance from a complicated design must be tempered by the fact that the effective retroreflector range varies only as the fourth root of the cross section. It's therefore imperative to maintain a reasonable retroreflector diameter, but striving for perfect optical performance may be a costly effort that does not merit the modest performance gain.

Several promising designs were developed for visible and mid-infrared applications, which embraced lessons learned in the standoff range study. These designs included a polymer-clad glass core approach for visible wavelengths and a concentric shell design using chalcogenide glass for the mid-infrared. Injection molding feasibility studies demonstrated that it was practical to fabricate visible polymer retroreflectors using this approach. Such an approach may be a practical solution for a related project at another national laboratory. Our primary focus is to develop mid-infrared designs; therefore compression molding of chalcogenide glass was adopted for fabricating mid-infrared retroreflectors. A significant effort was focused on developing practical compression molding methods that provided reasonable device yield. A custom compression molding press was developed in collaboration with a private engineering firm. Many successful molding studies were conducted during this effort, but ultimately the fragile nature of the chalcogenide glass and the unfavorable thermal expansion properties limited the feasibility of fabricating the thin shell structures needed for this design. An alternative Cat's Eye retroreflector design was therefore adopted. This design is constructed using two hemispherical structures that can be fabricated by conventional grinding and polishing methods or by molding with high yield. The cross section of this design is very good (and can be further improved by applying a highly reflective coating to the rear surface), but has reduced field of view as a design trade off as well as the isotropic nature of Luneburg-style retroreflectors.

\subsection{Step-Index Design}

Key to our design approach is approximating the ideal gradient index profile solution for an efficient retroreflector. The ideal index gradient has a starting index of 2.78 at the surface and 2.24 at the core, as shown in Figure 1 (left). In general, the index will tend towards a value of 2 at the center. The shape of the index profile will be concave if the starting index at the edge of sphere is greater than 2 and convex if the starting index is less than 2. We can construct a step-index approximation to this continuous profile using a commercial chalcogenide glass for the outer shell of a two-layer structure, for instance $\mathrm{Ge}_{30} \mathrm{As}_{13} \mathrm{Se}_{32} \mathrm{Te}_{25}$ (IG3) glass ${ }^{(1)}$ as the cladding and $\mathrm{ZnS}$ as the core with the clad and core having refractive indices of 2.787 and 2.200 , respectively, measured at $10 \mu \mathrm{m}$. We arbitrarily chose a core diameter of $5 \mathrm{~mm}$ because this size is readily commercially available for device prototyping. The design was optimized for maximum cross section (CS) by varying the thickness of the cladding while

(1) http://www.vitron.de 
minimizing the wavefront error merit function. The optimum CS was achieved when the design was optimized for an input beam diameter of $3.97 \mathrm{~mm}$. The final design culminated in a 1.372-mm-thick IG3 cladding surrounding a $5-\mathrm{mm} \mathrm{ZnS}$ core, which resulted in a total retroreflector diameter of $7.744 \mathrm{~mm}$. Plots showing the variation in SR and CS as a function of entrance pupil or input beam diameter are shown in Figure 2. A plot showing an ideal spherical gradient index profile with a superimposed stepindex profile, as well as a plot showing the step-index retroreflector design with ray trajectories at a pupil diameter of $3.97 \mathrm{~mm}$, is given in Figure 1. Note that because IG3 has an index of refraction of 2.787 at $10 \mu \mathrm{m}$, and surface reflectance of $22.3 \%$, the reflectance from the clad ball design taking into account front and rear surface reflections is approximately $13.4 \%$, or very nearly the optimum value of $14.8 \%$ shown in previous reports to be the theoretical maximum return for an optimally coated spherical retroreflector.
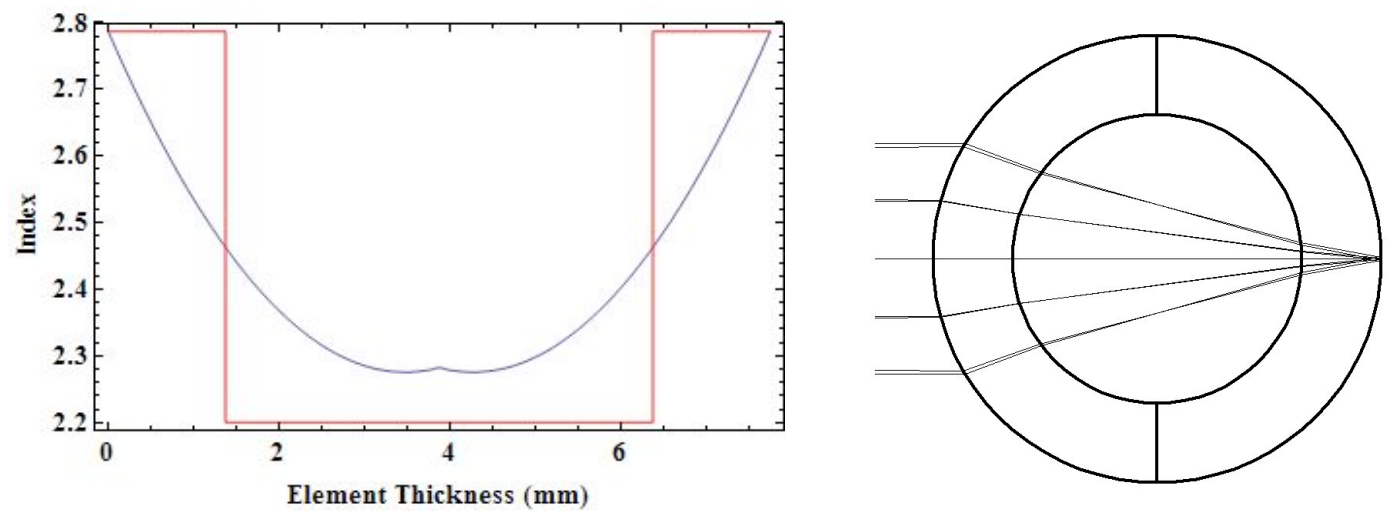

Figure 1. Comparison of the Step Index Profile and an Optimized Spherical Gradient Profile for a 7.744-mm-Diameter Sphere (left) and the Optimized Step-Index Retroreflector with Incident and Reflected Ray Trajectories (right)
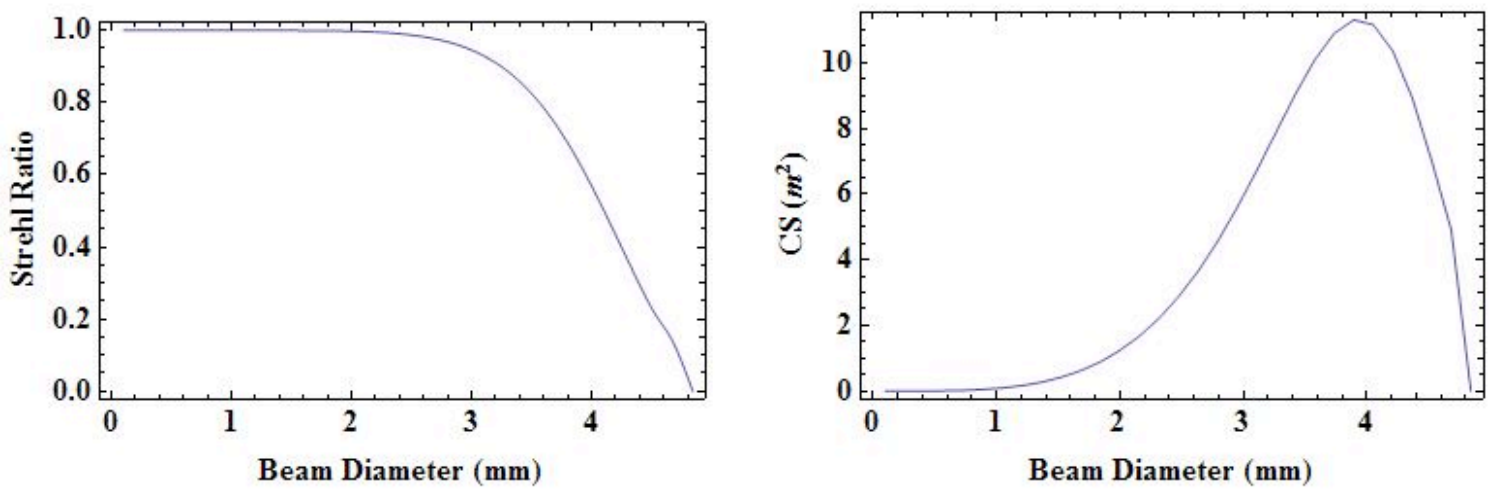

Figure 2. The SR Dependence as a Function of Entrance Pupil Diameter as the Clad Ball Design is Optimized (left), and the Dependence of SR on Entrance Pupil Diameter is Used to Compute the CS (right) that Reveals that the Peak CS is Achieved for an Entrance Pupil Diameter of $\sim 4 \mathrm{~mm}$. When the design is optimized for a beam diameter of $4 \mathrm{~mm}$, a SR of 0.61 results for the double-pass beam. 
Finally, it can be shown that the performance of a step-index clad ball design is nearly the same as that of the spherically symmetric gradient index solution over comparable input apertures and equal retroreflector diameters. The retroreflected beam SR as a function of increasing input beam diameter is shown in Figure 3 for three comparison designs: homogeneous $\mathrm{ZnS}$ and heavy metal oxide (HMOX) glass spheres, step-index sphere with IG3 cladding and ZnS core, and a spherical gradient index design. Because CS is proportional to the SR, one can see that the clad sphere predicts nearly the same performance as the superior, but practically unrealizable, gradient index solution. The relative CS performance is shown in Figure 4.

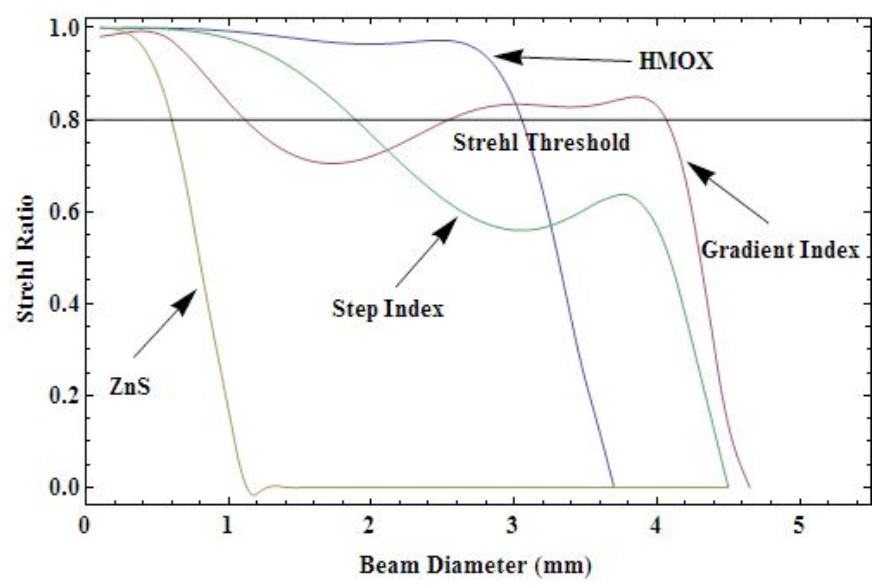

Figure 3. The Retroreflected Beam SR Versus Input Beam Diameter for Three Different Retroreflector Designs: Homogeneous Ball ( $\mathrm{ZnS}$ and HMOX glasses), Clad or Step-index Sphere, and Gradient Index Variants. Note that the clad sphere provides nearly the same performance as the ideal (and practically unrealizable) gradient index sphere. Also, the $\mathrm{n} \approx 2$ HMOX glass provides good performance over a wide aperture range.

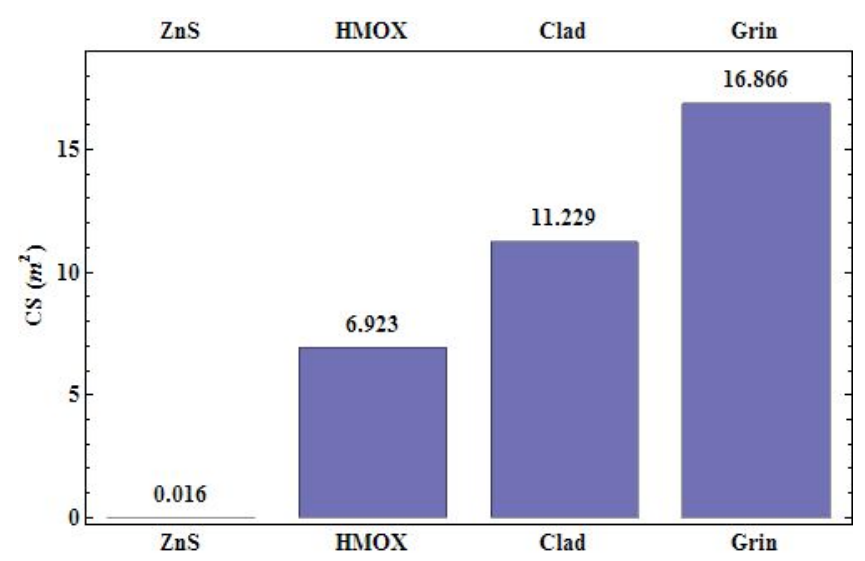

Figure 4. Cross Sections for the Strehl Ratios of the Four Designs Shown Earlier in Figure 3. The HMOX homogeneous ball with index of $\mathrm{n}=2$ provides reasonable return performance, but it displays only about half the CS of the clad ball design. 


\subsection{Cat's Eye Design}

A Cat's Eye retroreflector design was evaluated in our pursuit of an efficient and manufacturable miniature retroreflector. The Cat's Eye design is formed using two opposing optical hemispheres, with each having a prescribed optical index and radius of curvature that optimizes the retroreflection efficiency. In the case where the radii of the two hemispheres are equal, that solution reduces to the homogeneous index $n=2$ ball lens retroreflector. The Cat's Eye retroreflector design lends itself to traditional grind and polish optical fabrication methods, so in principle the manufacturability is scalable.

A mid-infrared Cat's Eye retroreflector design was developed using an 8-mm diameter Si hemisphere and a 4-mm Ge hemisphere, as shown in Figure 5 (left). The diameters and indices of these two commercially available hemispheres did not provide useful retroreflector properties, so the thickness of the Ge hemisphere was adjusted for optimum retroreflection efficiency. A hypo-hemisphere with nominal thickness of $1.941 \mathrm{~mm}$ was found to provide a retroreflector beam diameter of $\sim 3.3 \mathrm{~mm}$ that exceeded a SR of $80 \%$, as shown in Figure 5 (right).
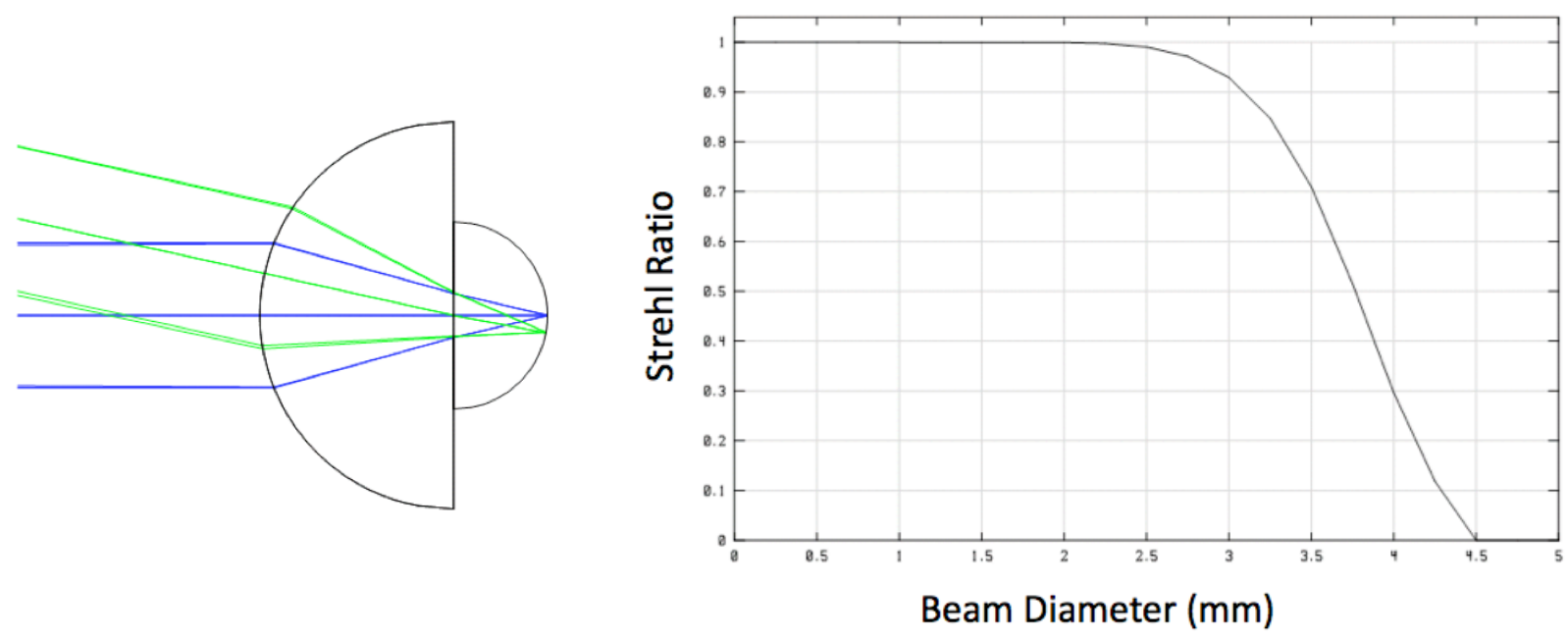

Figure 5. Cat's Eye Retroreflector Utilizing a Si and Ge Hemisphere (left) and SR versus Input Beam Diameter for the Cat's Eye Design (right)

Tolerance analysis was conducted on the Ge hemisphere thickness to optimize both the SR and the optical cross section performance with respect to the on-axis and off-axis regimes. The thickness was varied $1.941 \pm 0.01 \mathrm{~mm}$ and the input aperture was limited to $3.5 \mathrm{~mm}$ for this analysis. The SR is shown in Figure 6 for the on-axis and off-axis regimes. We see that the 10-micron thicker design provides the best performance in the on-axis regime, but has the lowest SR in the off-axis regime. The dependence on hemisphere thickness is greater in the off-axis regime, suggesting that a design with a thinner hemisphere thickness offers a better design choice. 

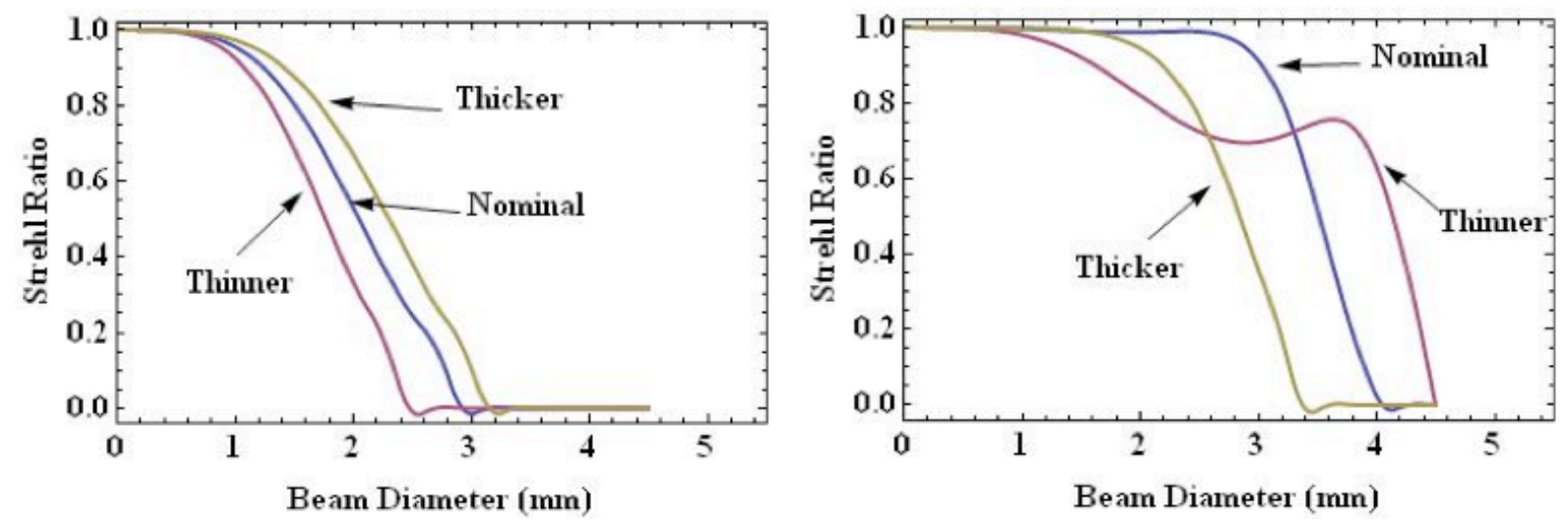

Figure 6. On-axis SR as a Function of Ge Hemisphere Thickness (left) and Off-axis SR as a Function of Ge Hemisphere Thickness (right)

Next the cross section was assessed as a function of Ge hemisphere thickness. The cross section has a strong dependence of hemisphere thickness for both the on-axis and off-axis regimes, as shown in Figure 7. The on-axis thinner hemisphere has a cross section 1.9 times larger than the nominal center thickness and 4.8 times larger than the thicker design, suggesting the thinner design superiority. In contrast the off-axis analysis showed that the thicker design has a cross section 2.8 times larger than the thinner design.
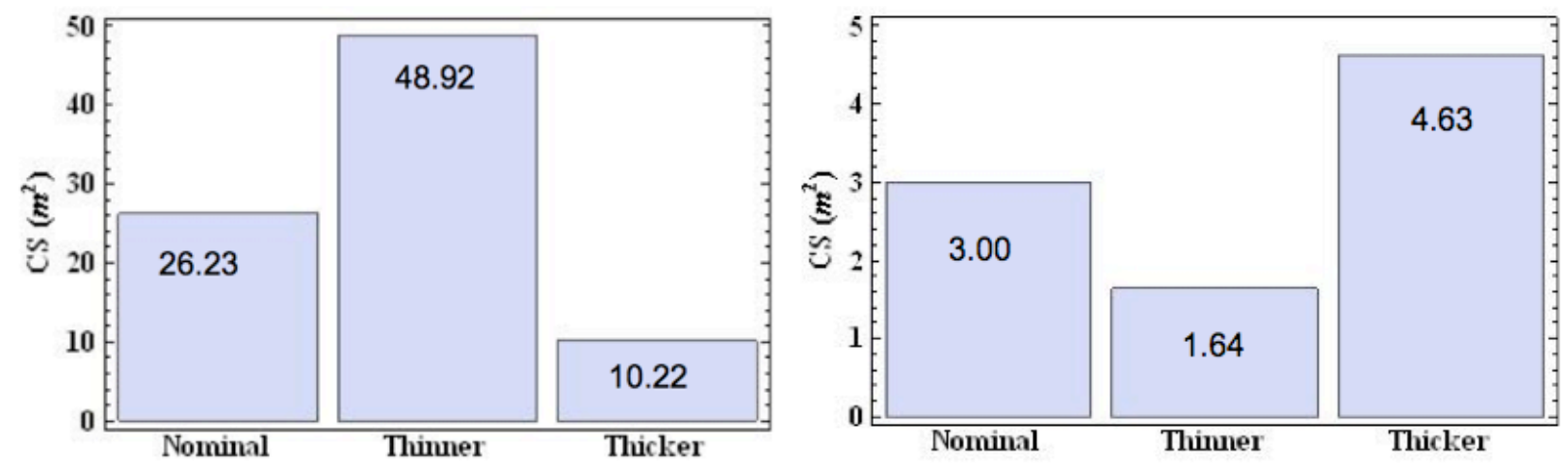

Figure 7. On-axis CS as a Function of Ge Hemisphere Thickness (left) and Off-axis CS as a Function of Ge Hemisphere Thickness (right)

Based on these modeling results, off-axis performance was sacrificed to maximize on-axis performance. The center thickness of the Ge hemisphere was specified at $1.931 \pm 0.01 \mathrm{~mm}$ for fabrication by a commercial optical component manufacturer. The curved Si hemisphere surface was also anti-reflection coated for the 5.3-micron test wavelength. After assembly the curved Ge surface was coated with a highly reflective gold layer using our sputter coater. The retroreflector is shown under test in Figure 8. 

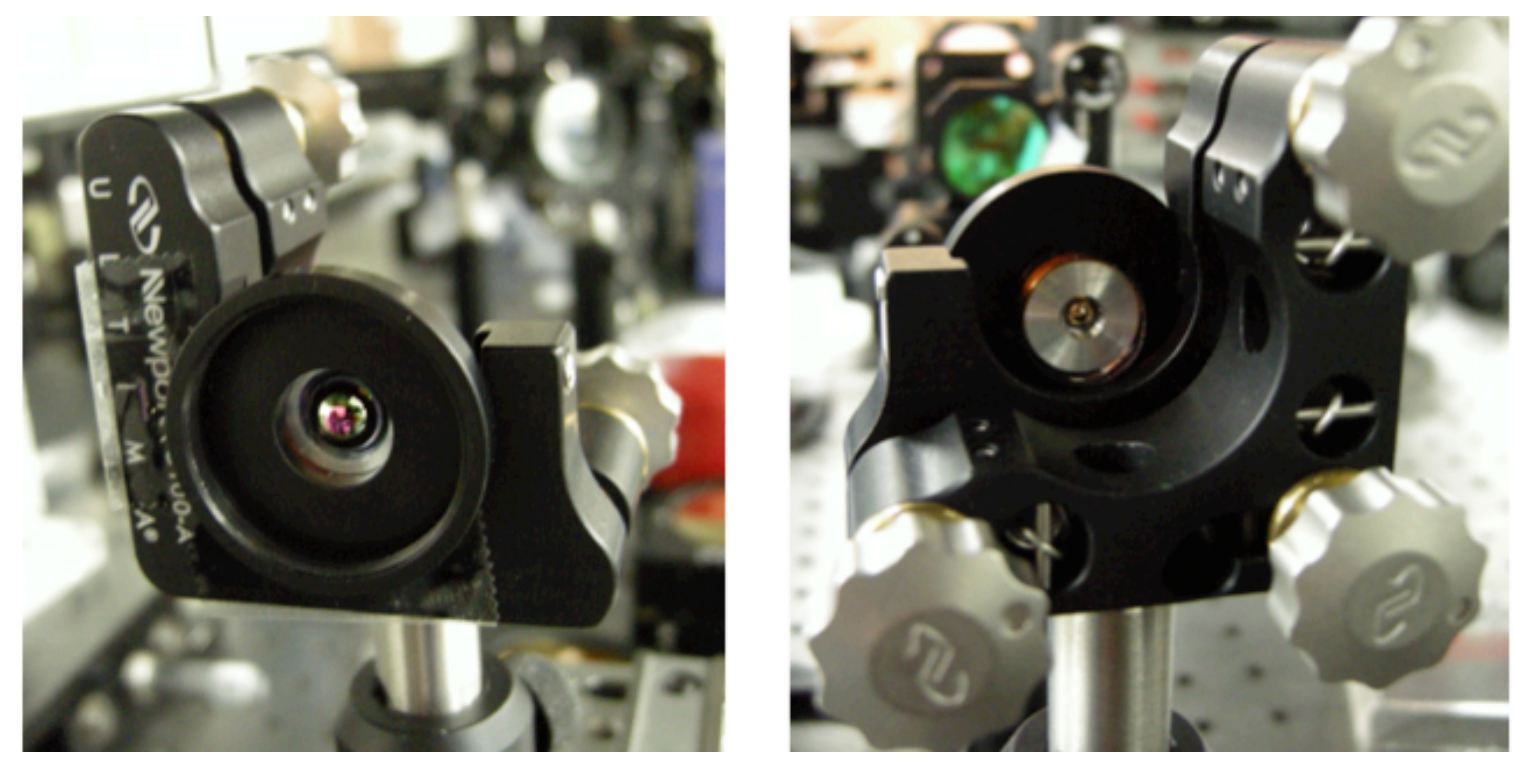

Figure 8. Anti-reflection Coated $8 \mathrm{~mm}$ Diameter Silicon Hemisphere that Serves as the Cat's Eye Input (left) and the Rear, Gold-coated $4 \mathrm{~mm}$ Diameter Germanium Hemisphere (right)

Lambda Research TracePro modeling software was used next to simulate the spatial and angular intensity distribution of laser light retroreflected at a 10-meter range, as shown in Figure 9. The retroreflected light has a sharp intensity lobe, with small spatial extent and angular spread. These results suggest that this mid-infrared Cat's Eye retroreflector design would provide very reasonable performance given the relatively simple design and assembly requirements.
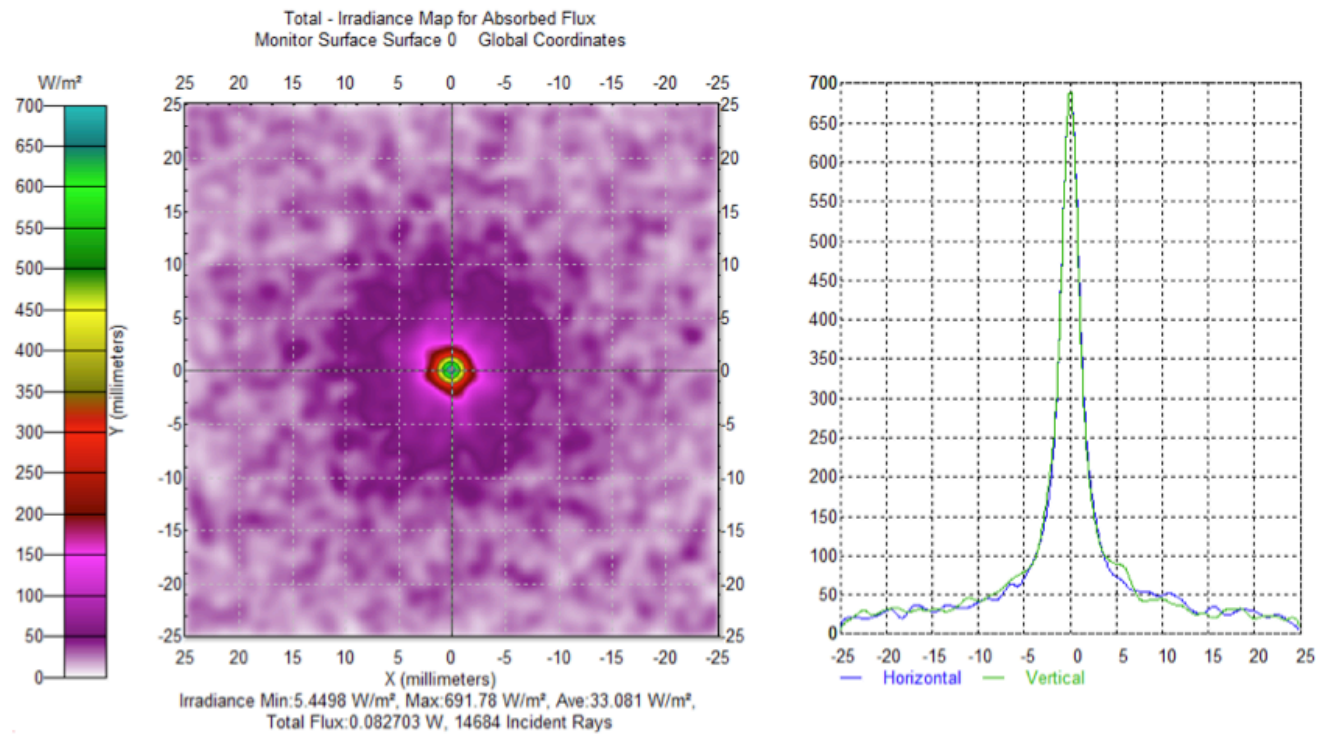

Figure 9. The Spatial Intensity Map (left) and the Angular Intensity Distribution of the Return at 10 Meters (right), Indicating Promising Cat's Eye Retroreflector Performance 
The assembled retroreflector, shown in Figure 8, was tested using a $5.3 \mu \mathrm{m}$ wavelength distributed feedback quantum cascade laser transmitter described previously (Anheier et al. 2009). The quantum cascade laser illuminated the retroreflector at varying range. A ferroelectric infrared camera recorded the retroreflected light through a beamsplitter placed in the beam path between the laser and retroreflector. Our measurements revealed that the retroreflected beam exhibited excessive beam divergence, causing the beam to expand quickly along the return beam path, as shown in Figure 10.
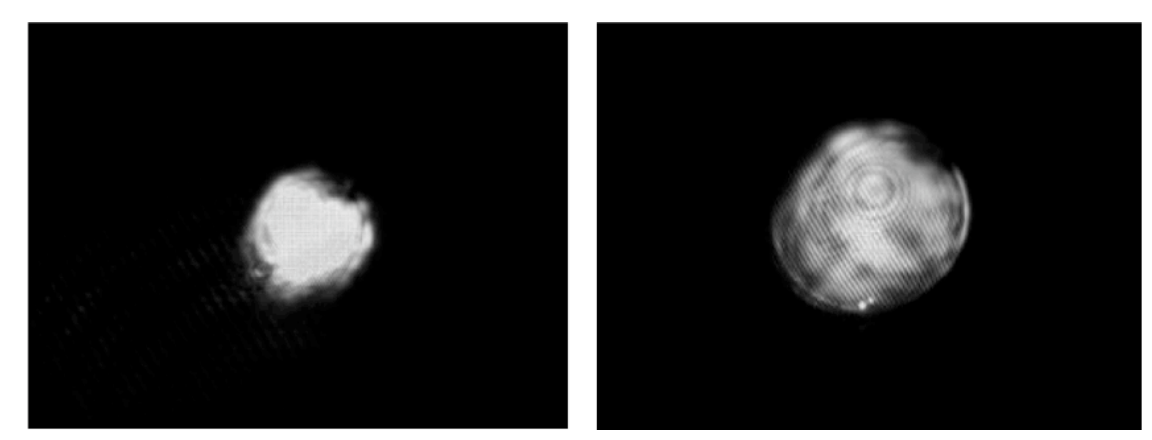

Figure 10. Cat's Eye Retroreflector Return at 0.6 Meters (left) and at 1.2 Meters (right). The observed fringe artifacts are generated by the protective window on the ferroelectric focal plane array.

It was obvious that this retroreflector assembly would not provide the performances suggested by Tracepro modeling shown in Figure 8. Further investigation revealed that the Ge hypo-hemisphere was misfabricated with $1.958 \mathrm{~mm}$ thickness, rather than the specified thickness $1.931 \pm 0.01 \mathrm{~mm}$. The additional Ge hemisphere thickness was found to be a crucial factor that reduced the ultimate potential of this Cat's Eye design. TracePro modeling software was again used to simulate the spatial and angular intensity distribution of laser light retroreflected from the misfabricated retroreflector. The results at 1-meter range are shown in Figure 11. The retroreflected light has a broad intensity lobe, with larger spatial extent and angular spread indicative of observed return beam divergence noted in Figure 10. While this defect prevented further evaluation at longer ranges, the Ge hypo-hemisphere element could be re-fabricated and tested, given additional time. 

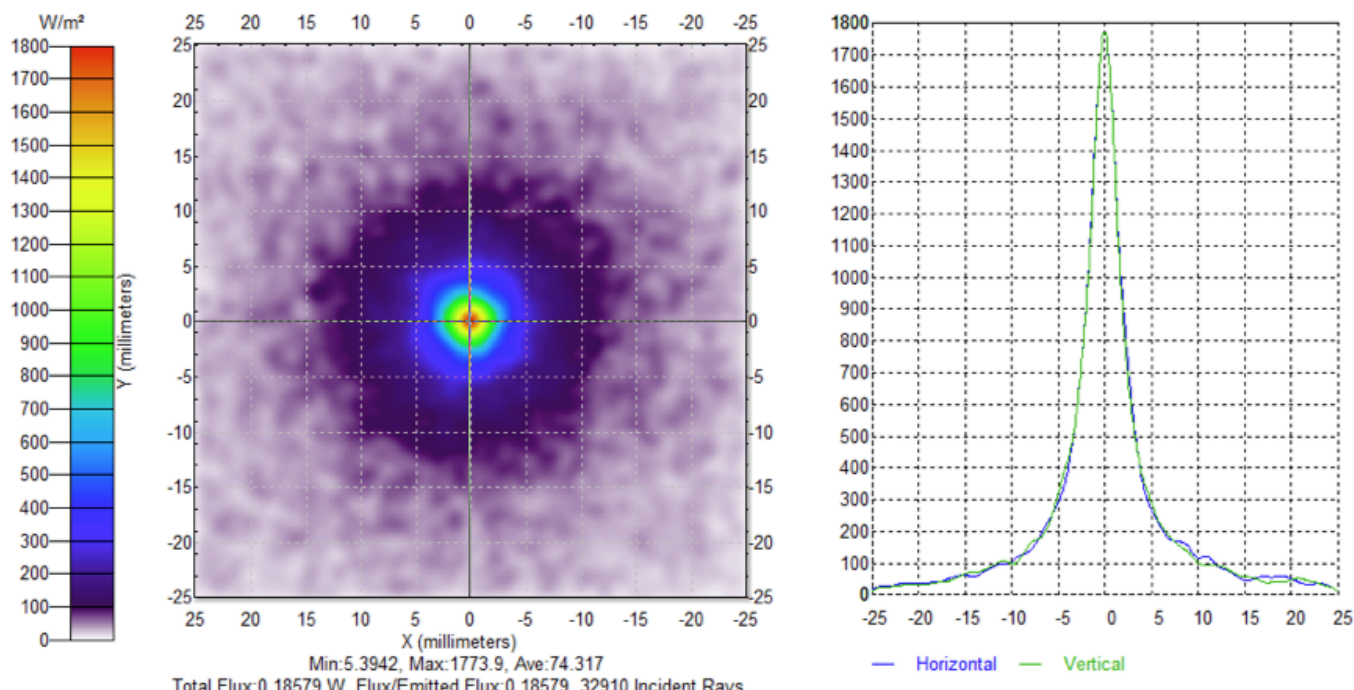

Figure 11. The Spatial Intensity Map (left) and the Angular Intensity Distribution of the Return at 1 Meter (right). The beam extent is noticeably larger than the ideal design shown in Figure 8.

\subsection{Retroreflector Sensing Layers}

\subsection{Nanocrystal Quantum Dot Materials}

Nanocrystalline semiconductor materials have unique material properties that make them attractive as sensing layers for the miniature retroreflector platform. Nanocrystal materials are comprised of 1- to 10 -nm wide structures formed from as few as 20 atoms. Nanocrystals made from luminescent materials are called quantum dots. The lattice structures of nanocrystals are smaller than the exciton-Bohr radius, causing the normal bulk continuum electronic structure to become discrete, as shown in Figure 12. This produces quantum confinement effects, dramatically increasing the luminescence brightness and reducing the emission spectral bandwidth $(\sim 30 \mathrm{~nm})$. The emission wavelength spans from the visible to the near infrared and the center wavelength is tunable based on the nanocrystal size. Quantum dots can be designed to provide stable, non-photobleaching emission, making them excellent candidates for extended deployment for remote sensing and tagging, tracking, and locating (TTL) applications. 

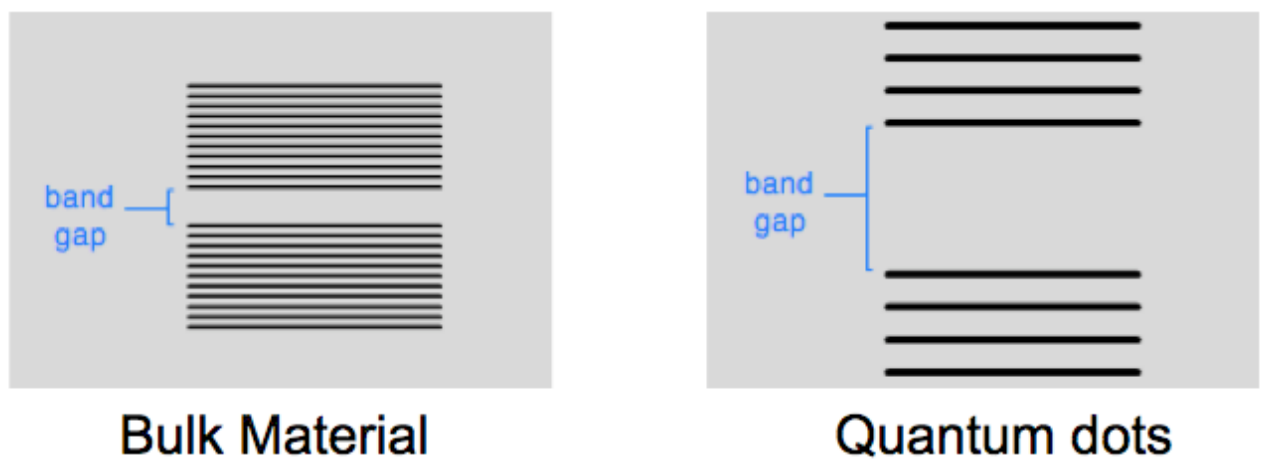

Figure 12. Lattice Structure of Bulk Materials that Leads to Broad Continuum Emission in Luminescent Materials (left) and Nanocrystal Quantum Confinement that Leads to Bright and Narrow Bandwidth Luminescence (right)

Index, $n=2$, optical glass (S-LAH79) ball retroreflectors were coated with quantum dots to test the feasibility of remote sensing using fluorescence- or luminescence-based sensing layers. The quantum dots are formed from $\mathrm{CdSe} / \mathrm{ZnS}$ nanocrystals and were commercially available from Evident Technology. The quantum dots were mixed in diluted UV-curable epoxy and spray-coated as a thin layer on the back side of the ball retroreflector. The dots were then immobilized by curing the epoxy using a UV lamp. The quantum-dot coated retroreflector was excited using a $5 \mathrm{~mW} 405 \mathrm{~nm}$ laser and the $605 \mathrm{~nm}$ luminescence emission was monitored using a $550 \mathrm{~nm}$ long-pass filter. The emission retroreflected back to the laser transmitter was found to be extremely intense. In a darkened test bed, the luminescence was easily observed by eye at a 46-meter standoff distance, as shown in Figure 13. The intensity fell quickly to zero when observing beyond 5 degrees off-axis, demonstrating promising retroreflector performance. It is likely that the quantum dot emission could be visible at several kilometers and during full daylight illumination if the laser excitation were modulated and the emission were observed synchronously. The coated retroreflectors were left unprotected on the laboratory bench for 6 months with no noticeable loss in luminescence emission intensity. Protected $\mathrm{ZnS}$ quantum dots can be synthesized and are available commercially. These quantum dots have emission at near-infrared wavelengths, making them particularly useful for TTL applications. 

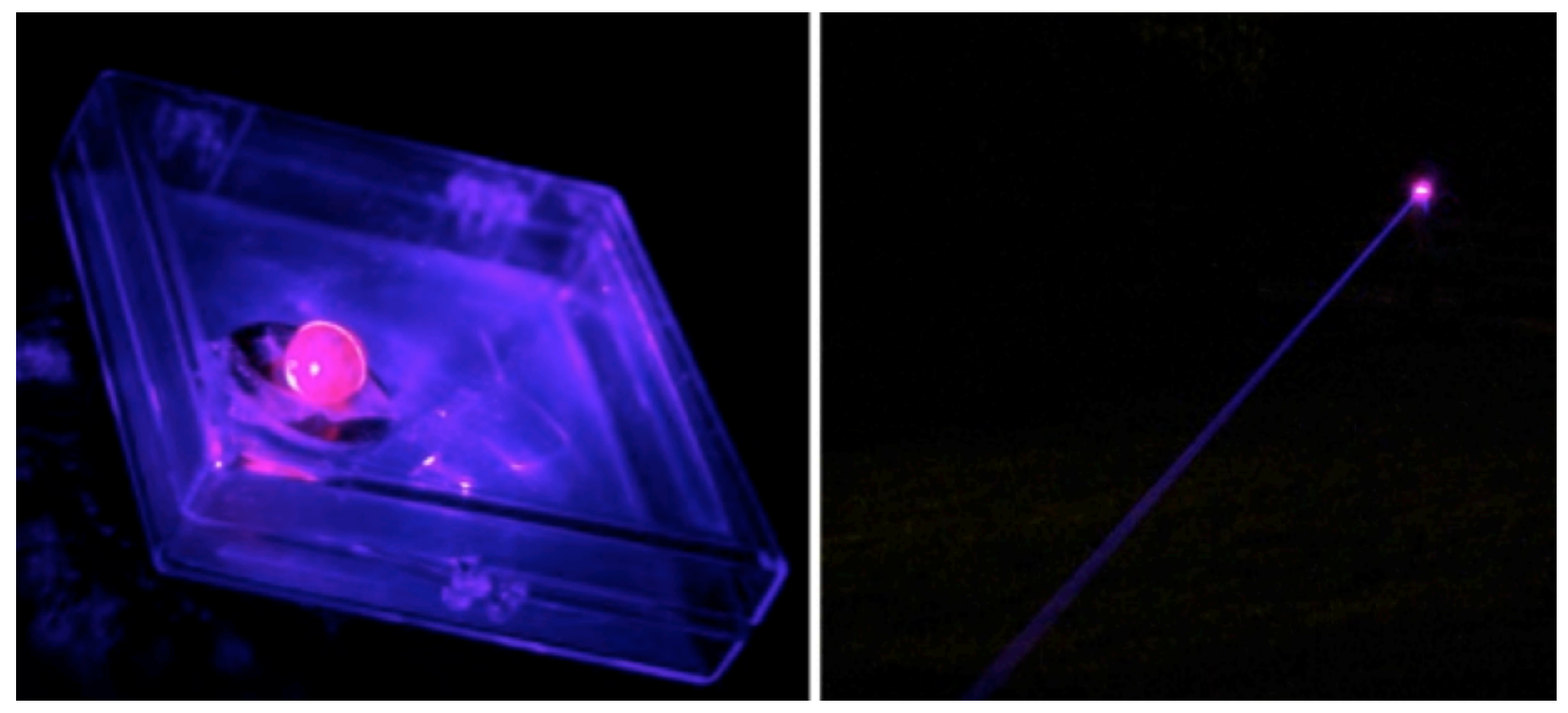

Figure 13. Homogeneous Index $n=2$ Spherical Retroreflector with an Applied CdSe/ZnS Quantum Luminescent Layer (left) and Extremely Strong Retroreflected Luminescent Emission Easily Seen about 46 Meters Away (right). The blue beam is the collinear $405 \mathrm{~nm}$ excitation laser and the central luminescent return beam.

Another novel quantum dot application is radiation detection. Optically stimulated luminescence (OSL) materials were developed at PNNL and now are commercially available for personnel protection radiation dosimetry. One PNNL-developed OSL material is $\mathrm{LiF}_{2}$. Bulk crystalline material is ground to micron dimension and dispersed into a polymer matrix, where it is then cast into dosimetry coupons. Cumulative dose is stored in the OSL in the form of color defect centers. After exposure to a radiation source, a blue light source is used to read out the luminescent proportion dose. The recorded dose is stable indefinitely and can be repeatedly read out, but the luminescence is broad and weak. This leads to the question whether $\mathrm{LiF}_{2}$ could be synthesized with nanocrystal dimension to form a radiation detection/monitoring thin film for the retroreflector platform.

A literature review was conducted to assess the feasibility of nanocrystal $\mathrm{LiF}_{2}$ fabrication that provides a narrow diameter distribution (the diameter determines the emission wavelength and broad diameter yields produce undesirable broad emission peak widths). The goal of this effort is to produce nanoscale materials that exhibit efficient radiation-induced color-center defect formation that can be read out by remote optical interrogation using the miniature retroreflector platform. The literature search returned very little information on the synthesis and properties of $\mathrm{LiF}_{2}$ nanoscale materials. $\mathrm{LiF}_{2}$ nanoparticles have been synthesized using a few different preparation methods, including: precipitation reactions with $\mathrm{LiOH}$ and $\mathrm{HF}$; co-precipitation of $\mathrm{LiCl}$ and $\mathrm{NH}_{4} \mathrm{~F}$; and microemulsion. The size and shape of the resultant material is dependent on the method used. The second method, employing the $\mathrm{LiCl}$ and $\mathrm{NH}_{4} \mathrm{~F}$ precursors, offers the most benefits in that it has the greatest potential for simple scale-up, and the reagents are the least hazardous of all the published methods. This method also has some key data on the effects of various dopants on thermoluminescent properties in the nanoscale. The smallest core size particles reported use the microemulsion method, with a core diameter of $\sim 20 \mathrm{~nm}$. This method is time intensive and generates significant solvent waste, and product yield is generally poor. In addition, it is 
unknown whether the synthesis conditions can be tuned to yield the desired nanocrystal dimension smaller than the exciton Bohr radius. Numerical calculations conducted to determine the exciton Bohr radius for $\mathrm{LiF}_{2}$ suggest a core diameter around $1 \mathrm{~nm}$ is needed to realize the desirable quantum confinement. Given this and the facts that the synthesis process is difficult and the diameter distributions are large, we have decided that this investigation would be better suited for a future project rather than one near its end.

\subsection{Conclusions}

Design concepts and fabrication methods to produce miniature infrared spherical retroreflectors have been investigated during the course of this project, including comparative design and modeling results for homogeneous, gradient index, and step-index full- and half-clad and Cat's Eye designs. The calculated CS values were used to determine the normalized standoff detection range potential for each retroreflector design. The analysis results showed, for example, that the step-index design's 33\% CS reduction (as compared to the spherical gradient-index design) led to a standoff range reduction of only $10 \%$. This is a significant result and it should serve to temper ambitions to develop spherical gradient fabrication methods if they become costly endeavors or if a $90 \%$ solution (step-index) or $80 \%$ solution (single-index HMOX glass) will satisfy the deployment requirements.

A final mid-infrared retroreflector design based on a Cat's Eye design was considered. Custom optical components were fabricated by a commercial optical manufacture. The Cat's Eye retroreflector was assembled and tested in our lab. The retroreflector sensing task evaluated retroreflector sensing films based on nanocrystalline materials called quantum dots. To test the feasibility of this concept, ball retroreflectors of index $n=2$ were coated with quantum dots. In a darkened laboratory, the laser-excited photoluminescence was easily observed by eye at a 46-meter standoff distance. Quantum dots can be designed to provide stable, non-photobleaching emission, making them excellent candidates for extended deployment for remote sensing and TTL applications.

A simple, low-cost solution for mass production of infrared spherical retroreflectors remains elusive. PNNL identified the Flow Focused micro-nozzle early in the project as having the best potential for commercial production of miniature retroreflectors, yet prohibitively large investments were required to realize this approach. A less risky compression-molding approach was adopted, but conflicting material properties prevented decisive closure on the fabrication bottleneck. While the fabrication focus was not fully satisfied, PNNL made major contributions to this field by developing practical and efficient optical designs and an analysis formalism that clearly illustrates the relative performance and provides design rules and performance metrics to objectively compare competing designs. We anticipate this body of work will stimulate future research in this area and lead to enabling technology for remote sensing applications. 


\subsection{References}

Anheier NC, Jr, BE Bernacki, BR Johnson, BJ Riley, and WA Sliger. 2005. FY 2005 Miniature Spherical Retroreflectors Final Report. PNNL-15577, Pacific Northwest National Laboratory, Richland, Washington.

Anheier NC, Jr, BE Bernacki, and K Krishnaswami. 2006. FY 2006 Miniature Spherical Retroreflectors Final Report. PNNL-16320, Pacific Northwest National Laboratory, Richland, Washington.

Anheier NC, Jr, BE Bernacki, and K Krishnaswami. 2008. FY 2007 Miniature Spherical Retroreflectors Final Report. PNNL-17287, Pacific Northwest National Laboratory, Richland, Washington.

Anheier NC, Jr, BE Bernacki, NA Klymyshyn, K Krishnaswami, and CP Rodriguez. 2009. FY 2008 Miniature Spherical Retroreflectors - Final Report. PNNL-18344, Pacific Northwest National Laboratory, Richland, Washington.

Anheier NC, Jr, BK Hatchell, KL Gervais, MD Wojcik, K Krishnaswami, and BE Bernacki. 2009. "Compact Quantum Cascade Laser Transmitter." In Laser Technology for Defense and Security V, vol. 7325, ed. Mark Dubinskii, Stephen G. Post, p. Art. No. 73250F. Society of Photo-Optical Instrumentation Engineers, Bellingham, Washington. doi:10.1117/12.818034. 


\section{Distribution}

No. of

Copies

1 Dr. Victoria T. Franques

U.S. Department of Energy

NNSA/NA-22

1000 Independence Ave. SW

Washington, DC 20585

1 Dr. Rhys M. Williams

U.S. Department of Energy

NNSA/NA-22

1000 Independence Ave. SW

Washington, DC 20585

1 Mr. Eric Sanders

U.S. Department of Energy

NNSA/NA-22

1000 Independence Ave. SW

Washington, DC 20585

1 Dr. Vaughn Stanley

U.S. Department of Energy

NNSA/NA-22

1000 Independence Ave. SW

Washington, DC 20585
No. of

Copies

10 Internal Distribution

Pacific Northwest National Laboratory

P.O. Box 999

Richland, WA 99352

Anheier, NC

K5-25

Bernacki, BE

K5-25

Qiao, HA

K5-25

Sharpe, SW

$\mathrm{K} 8-88$

Watkins, ML

K5-25

Information Release (2)

K1-06 


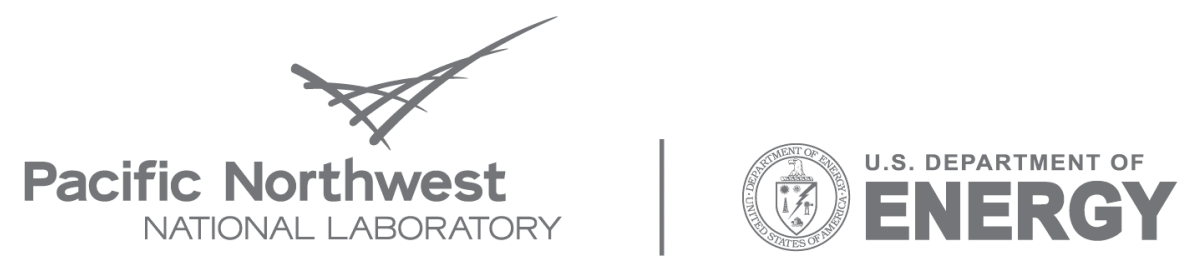

Proudly Operated by Battelle Since 1965

902 Battelle Boulevard

P.O. Box 999

Richland, WA 99352

1-888-375-PNNL (7665)

www.pnl.gov 